An Overview of the CAPITL Software Development Environment

Paul Adams

Marvin Solomon

Technical Report \#1143

April 1993 



\title{
An Overview of the CAPITL Software Development Environment ${ }^{*}$
}

\author{
Paul Adams \\ adams@cs.wisc.edu \\ and \\ Marvin Solomon \\ solomon@cs.wisc.edu
}

The CAPITL programming environment is comprised of a shared, object-oriented, versioned database, an embedded logic-based data-manipulation language, and a graphical user interface. With each software object the database stores a rich set of atributes that describe its syntax, intended semantics, and relationship to other objects. CAPITL is implemented in POL, a data model and deductive query language with elements of persistent, object-oriented and logicbased programming languages. POL is implemented in and tightly coupled with $\mathrm{C}+\mathrm{H}$.

A request for a derived object consists of a partial description of its attributes. A planner written in POL searches the database for tools and sources that can be combined to create an object meeting the description. Since tools are stored in the database like other objects, plans that create tools as well as intermediate inputs are possible. A builder, also written in POL, executes plans to materialize software products. The builder verifies that existing objects are current, minimally re-applying tools as sources, tools, or system descriptions change.

After an overview of the database and the POL programming system, we outline CAPITL's logic-based approach to system modelling, illustrating it with two examples. We conclude with a status report and an outline of future directions.

\section{Introduction}

Large software systems are hard to build and maintain. The sheer number of components involved make the management, coordination, and storage of the components difficult. Because of the malleable nature of software, components are constantly changing. Change is not limited to source text; attributes of the source files, relationships among them, tools used to process them, and even architectures of whole subsystems change as bugs are fixed, new functionality is added, and components are re-organized. Maintaining invariants in an evolving system is a critical task for any support system $[22,27,30]$.

The CAPITL ${ }^{1}$ project at the University of Wisconsin has been investigating a logic-based approach to software configuration management [21]. The basic thesis of our approach is that if all objects in the

"This work was supported in part by the Defense Advanced Research Projects Agency under ARPA Order No. 8856 (monitored by the Office of Naval Research under contract N00014-92-J-1937).

To appear in the Proceedings of the Fourth International Workshop on Software Configuration Management.

Authors' address: Computer Sciences Department, University of Wisconsin-Madison, 1210 W. Dayton St., Madison, WI 53706.

'Computer Aided Programming In The Large 
environment carry with them sufficiently detailed descriptions, desired software products can be described declaratively and the system can infer the process necessary to build them. To test these ideas, we have constructed a environment that tightly integrates a logic-based language with a versioned, object-oriented database. By tightly coupling the database with both an imperative object-oriented language and a declarative language, CAPITL gets the best of both worlds: declarative queries and specifications, and objectoriented extensibility and state encapsulation. This paper describes the main components of the environment and outlines how they support maintenance of large software systems.

CAPITL was designed with four principles in mind.

- Uniformity. All objects are represented and described in a common language.

- Locality. The information that describes an object and its relationships with other objects is directly associated with that object.

- Extensibility. New types of objects, new descriptive properties, and new relationships can be added easily.

- Flexibility. Policies for access control and modification to objects can be specified rather than such policies being "wired in."

CAPITL consists of three main components: a shared, versioned database, a graphical user interface, and a fully embedded logic-based data-manipulation language. The CAPITL database records all aspects of software construction: source files, documentation, sub-systems, system descriptions, tools, executables, and configurations. The form and function of each database object, as well as its relation to other objects, is described in detail by attributes stored with it. Support for efficient maintenance of multiple versions of the database is built in. A compatibility feature allows existing Unix tools to manifulate CAPITL objects as if they were Unix files.

The database is accessible via an interactive browser/editor based on the X Window System [25] and the InterViews graphical toolkit [17]. CAPITL's browser can navigate the version history of the database and the links between objects. It also provides facilities for the display and manual update of objects.

Most of the features of CAPITL are implemented in POL [2], a data model and deductive query language synthesized from elements of persistent, object-oriented, and logic-based programming languages. POL is tightly integrated with the database (all database objects are POL terms) and with a general-purpose host language $(\mathrm{C}++)$. The database uses the Exodus toolkit [3] to provide low-level concurrency control, error recovery, and network access. POL includes a logic-based programming language called Congress, which also servers as a query and update language.

CAPITL uses Congress as the basis for a tool that automatically builds and maintains derived objects. An architect of a software system describes tools and policies using Congress as a specification language. A program written in Congress accepts declarative specifications of desired products and deduces plans to locate or construct them. CAPITL thus provides a platform supporting applicationspecific notions of consistency and correctness.

The remainder of this paper is organized as follows. Section 2 describes the POL data model, the embedded language Congress, and the interface between Congress, $\mathrm{C}++$, and the daabase. The use of the database to store software objects is explored in Section 3. Section 4 explains how CAPITL is used for software configuration management (SCM). Section 5 illustrates these ideas with two concrete examples. Section 6 discusses related work. We close with a status report and future plans. 


\section{POL}

POL (Persistent Objects with Logic) is a mixture of three styles of programming language: objectoriented, logic-based, and persistent. Each style has features that make solving certain problems easier: Object-oriented languages encapsulate state and behavior and support extension by inheritance; logic programming languages allow programmers to concentrate on describing what a solution is rather than how to find it; persistent programming languages relieve the programmer of the burden of saving and restoring data. By combining features from all three domains, POL provides an environment in which application programmers can take advantage of the particular style that best suits the problem at hand.

POL derives its object-oriented features from $\mathrm{C}++(\$ 2.2)$, persistence from the Exodus database toolkit (\$2.3), and logic-based features from Congress-a derivative of Prolog (\$2.4). POL integrates these components with a shared data model and a two-way embedding of Congress in $\mathrm{C}++$ and $\mathrm{C}++$ in Congress. The remainder of this section describes the data model, the three components, and the interfaces between them.

\subsection{Term Space}

As in Prolog and LISP, POL uses one data structure for both programs and data. A term space is a directed graph with labelled nodes and arcs. The label associated with a node is called its functor ${ }^{2}$ and the label associated with an arc is called its selector. No two arcs leaving the same node may have the same selector. A term is the subgraph of the term space reachable from a node, called the root of the term. We occasionally identify a term with its root node, when the meaning is clear from context. For example, the "functor of a term" means the functor of its root node.

POL is "identity-based": Two nodes with identical contents are nonetheless considered to be distinct. Nodes are explicitly created, and updates to a node do not change the node's identity. In this way POL differs from "value-based" Prolog and relational databases, and more closely resembles so-called "objectoriented" databases.

POL supports multiple versions of the term space called worlds, and uses an algorithm devised by Driscol et. al. [5] that supports efficient "checkpointing" of the entire term space. POL has operations to save the current term space as a world, and to reset its state to any previously saved world. A checkpoint operation does not copy the entire term space, but only an amount of data proportional to the changes made since the previous checkpoint.

\section{2. $\mathrm{C}++$}

$\mathrm{C}+$ is a strongly typed object-oriented language derived from $\mathrm{C}$. $\mathrm{C}++$ classes encapsulate both data and operations on that data. $\mathrm{C}++$ allows multiple inheritance and supports information hiding via explicit public/private declarations. Subclasses can override methods of their super-class as well as add new data fields and operations.

$\mathrm{C}++$ classes are used in POL to provide a concrete realization of term space nodes and arcs. Data structures used to represent nodes come in a variety of flavors. Leaf nodes (nodes with no outgoing arcs) are classified according to the data types of their functors: integers, real numbers, printable strings, byte strings (arbitrary binary data) or "variables." (Variables are explained in Section 2.4.) An internal node contains a functor (which must be a printable string) and a table of references to other nodes indexed by

\footnotetext{
${ }^{2}$ This unfortunate choice of terminology is inherited from Prolog.
} 
distinct printable strings. Internal nodes are similar to $\mathrm{C}$ structs, Pascal records, SNOBOL tables, and AWK associative arrays. Unlike structs or records, the number and names of "fields" may vary dynamically, and their contents are restricted to be non-null pointers to nodes. $\mathrm{C}++$ subclass derivation is used to add additional behavior and restrictions to classes of internal nodes. We shall return to this point in Section 3.

\subsection{Exodus}

Exodus [3] is a toolkit for creating custom database systems. POL uses two components of Exodus, a low-level storage subsystem and a persistent programming language. The Exodus Storage Manager provides efficient access to an arbitrary-sized persistent chunk of uninterpreted data called a "storage object" through a unique identifier called an "OID." The Storage Manager supports concurrency control through two-phase locking, and a simple transaction facility with full recovery from hardware and software failures. The E programming language [23] is an extension of $\mathrm{C}++$ that supports persistent data-data that retains its state between runs of a program. E syntax extends $\mathrm{C}++$ with a "db" version of each primitive type and type constructor (e.g. dbint, dbclass $1 \ldots$, etc.). Instances of a db type can be allocated from a persistent heap. The E runtime support library ensures that persistent data structures are securely stored on disk at the end of a transaction, and are fetched on demand (whenever a pointer to one is dereferenced). POL implements the term space with persistent data structures.

Throughout this paper, all references to the $\mathrm{C}++$ programming language should be understood as referring to the E dialect of $\mathrm{C}++$.

\subsection{Congress}

Congress may be described as a logic programming language, a deductive database query language, an embedded query language, or a library of classes for convenient database access, depending on one's point of view. Since Congress is implemented as a library of classes, any $\mathrm{C}++$ program can use Congress as a "higher level" alternative to or enhancement of the raw $\mathrm{C}++$ term interface.

As a logic-programming language, Congress is a dialect of LOGIN [1], an extension of Prolog that supports cyclic terms. It provides transparent persistence, and has an identity-based rather than valuebased semantics. The following paragraphs briefly describe the syntax and semantics of Congress. The reader who is familiar with logic programming may skim this section.

Congress programs are built from terms in the POL term space. A program is a set of procedures, a procedure is a sequence of clauses, and a clause is a sequence of terms. In particular, a clause consists of a single term called its head and a sequence of zero or more additional terms called its body. The predicate of a clause is the functor of the root node of its head term. A procedure is a sequence of clauses with a common predicate, referred to as the name of the procedure. A program is a set of procedures with distinct names.

The operational behavior of Congress is defined by the same recursive backtracking search as in Prolog. A goal (or "query") consists of a term. It is "called" ("evaluated," "proved") by searching the procedure named by its functor for a clause whose head "matches" the goal. If a maiching clause is found, each term in its body is called in turn. If no matching clause can be found, the interpreter backs up by undoing all of its actions since the last "choice point" (the point at which a clause was chosen to match against a goal) and attempts another match. The process continues either until all goals and subgoals have been proven, in which case the original call "succeeds," or until all alternatives have been exhausted, in which case it "fails". 
The heart of this process is the definition of "matching" between terms, called unification. ${ }^{3}$ Congress uses a variant of unification that supports cyclic terms [1]. The goal of unification is to determine if two terms are isomorphic, or can be made isomorphic by substituting terms for variables. Two terms unify if their roots match (have the same functor) and corresponding successors (recursively) unify. That is, if both roots have arcs with the same selector leaving them, the nodes reached by these arcs must also unify. As mentioned in $\$ 2.2$, some nodes are designated as variables; a variable matches any node. A side effect of a successful unification is an equivalence relation that records which nodes were matched. The evaluation of a call adds a copy of a clause to the term space and identifies nodes matched as a result of unifying its head with the query. The terms of the body are called in this extended term space.

Congress has a character-string expression language that may be used to enter or print programs or fragments of programs, or to enter queries from the keyboard. $A$ term $t$ may be denoted $f\left(s_{1} \Rightarrow t_{1}, \cdots, s_{n} \Rightarrow t_{n}\right)$, where $f$ is its functor, $s_{1}, \cdots, s_{n}$ are the selectors of the arcs with $f$ as their tail, and $t_{l}, \cdots, t_{n}$ are textual representations of the terms at the heads of the corresponding arcs. A variable is denoted "e." A tag (an alphanumeric string starting with a capital letter) is used to indicate shared subtrees or cycles. For example, the term

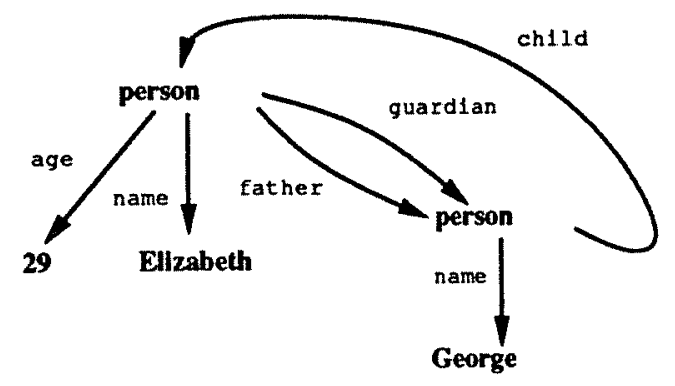

may be denoted ${ }^{4}$

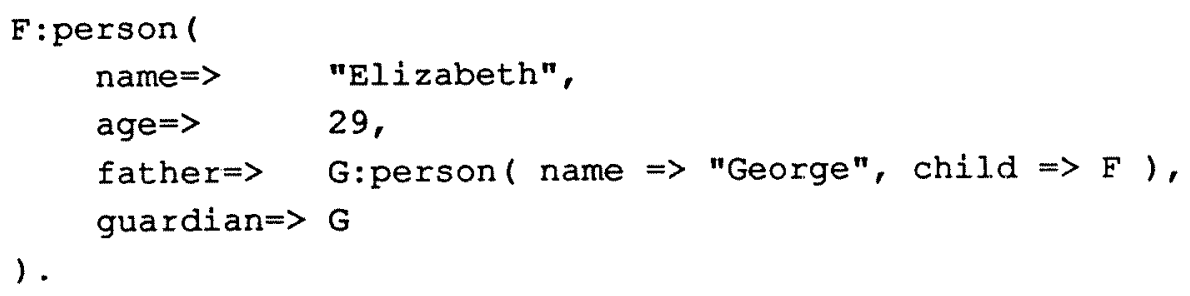

The expression language denotes a clause with head $t_{0}$ and body $t_{1}, \ldots, t_{n}$ as " $t_{0}:-t_{1}, \ldots, t_{n}$." The expression language also includes "syntactic sugar" for representing common infix operators such as $+, \star,-, /$, and for Prolog style lists. For example, the expression $[a, b$, Tail) denotes the same term as the expression cons (car $\Rightarrow a, c d r \Rightarrow$ cons (car $\Rightarrow b$, $\mathrm{cdr} \Rightarrow \mathrm{Tail}$ )). A missing selector implies an edge labelled with an integer and occurrences of $\mathbb{a}$ can be omitted in most cases. For example, $f(a, x)$ is the same as $f(1 \Rightarrow a, 2=>x: @)$.

${ }^{3}$ Background material on unification can be found in many logic programming texts and in an excellent survey by Knight [13].

AA functor that contains non-alphanumeric characters or starts with an upper-case letter must be quoted. 
With these abbreviations, the Congress expression language becomes a strict superset of Prolog. It extends Prolog in two important ways. First, the successors of a node are indicated by keyword rather than positional notation. This extension helps avoid programming errors. ${ }^{5}$ For example, the Congress expression employee (age $=>25$, salary $=>30$ ) is less confusing than the corresponding Prolog expression employee $(25,30)$. Second, while Prolog terms are trees (except for identification of multiple occurrences of the same variable), Congress allows arbitrary graphs, including cycles. Variables serve two purposes in Prolog: They represent "wild cards" for pattern matching and they indicate sharing. The expression language of Congress uses the functor "@" for the first purpose and tags for the second.

\subsection{Embedding}

The coupling between $\mathrm{C}++$ and Congress is a two-way embedding: Each language appears to be an embedded sub-language [20] of the other. Each language retains its own style. The embedding does not alter the syntax or semantics of either language. Since Congress programs are $\mathrm{C}++$ data structures, a $\mathrm{C}++$ program can construct or modify a program and call the Congress interpreter to execute it, capturing all output as $\mathrm{C}++$ structures. The embedding is bidirectional: $\mathrm{C}++$ procedures can be declared as external predicates in Congress. When the interpreter encounters a goal whose functor is an external predicate, it calls the corresponding $\mathrm{C}++$ procedure, passing it the goal and a description of the current state of the computation (added nodes and bindings). The procedure may make any modifications to the environment it deems appropriate (for example, adding bindings) and return either a success or failure indication. During backtracking, the interpreter may call the procedure again, asking whether it can succeed in other ways. In short, an external predicate is any $\mathrm{C}++$ procedure that follows the protocol of a Congress procedure.

External predicates have proven extremely useful. They are used to implement all of the built-in predicates usually found in Prolog implementations, such as arithmetic operations, as well as other functions that are awkward or impossible to implement directly in Congress, such as file system access or invocation of other programs. The Congress interpreter is itself an external predicate so Congress programs can invoke the interpreter recursively.

\section{CAPITL Object-base}

CAPITL uses the persistent term space of POL to build an object-oriented database (or object-base for short). All the entities used during the process of software development-source files, derived binaries, documentation, executable tools, and descriptions of subsystems--reside in the object-base. Properties attached to each object describe it and its relationship to other objects. The object-base is organized into a tree-structured naming hierarchy similar to a Unix file system (\$3.1). The object-base can be accessed interactively, from programs written in $\mathrm{C}++$ or Congress, or through a Unix compatibility feature (\$3.3.2). In the last case, an extension to Unix path-name syntax provides access to versions of the term space.

\subsection{Objects}

The fundamental entity in the CAPITL database is the object. An object is a "heavier weight" term that is guaranteed to have certain selectors with built-in semantics. Viewed from the Congress language, the term space is simply a labelled directed graph. Viewed from $\mathrm{C}++$, the nodes of the graph are further classified into an inheritance hierarchy. As explained in $\$ 2.2$, the first level of the hierarchy separates nodes into leaf nodes (which are further classified as integers, byte strings, etc.) and intemal nodes, which

SI also has a rather subtle effect on the definition of unification. See the LOGIN paper [1] for details. 
contain pointers to other nodes. Among internal nodes, CAPITL further designates some as object nodes, which are guaranteed to have certain selectors. If $o$ is an object and $s$ is one of its selectors, the $s$ attribute of $o$ is the term referenced by selector $s$ in $o$. Although attributes of objects behave just like other selectors from the Congress interface, they are generally implemented by special-case code. All objects have integer attributes owner, group, permissions, mtime, atime, and ctime, interpreted as in Unix. They also have three other attributes, contents, narne, and di rectory described below.

Sometimes descriptive information about an object is not static, but rather is a function of other attributes of the object. For example, a text document may have an attribute, imports, that lists any figures that it imports. Given a tool that scans the text of a document and computes the list of figures, the value of the imports attribute could be specified as a function of the tool applied to the contents attribute. In CAPITL such attributes are called derived attributes. The value of a derived attribute is not represented directly as a term, but as a function application, so that CAPITL can recompute the value when necessary. The only derived attribute currently supported is the contents atribute of an object with a non-empty provenance attribute (see $\$ 4$ for more detail on these attributes). We are investigating a more general mechanism to declare derived attributes and the functions that compute them.

Objects are further classified as directories, files, and symbolic links. A directory object is similar to a Unix file-system directory. Its contents attribute is a list (constructed of cons nodes) of references to other objects. The directories create a tree-structured name space similar to the Unix file system.

File objects are further classified as plain, delta, term, and composite. The contents of a plain file object is a byte-string atom. It has exactly the same semantics as a Unix "plain" file (see 83.3.2). Delta files have additional operations to "compress" and "uncompress" their contents. Delta files represent consecutive versions of their contents as delta lists using an algorithm similar to RCS [29]. The contents of a term file is an arbitrary Congress term. A composite file, like a directory, contains a list of references to other objects, but it does not emulate all the behavior of a Unix directory, nor is it constrained to be part of a strict tree structure.

Finally, a symbolic link object's contents is a printable-string atom. It is singled out as a special kind of object to support the Unix compatibility interface (\$3.3.2).

\subsection{Versions}

CAPITL uses the world mechanism of POL to maintain multiple snapshots of the database. Each operation accessing the CAPITL database is done in the context of a designated current world, and any changes made by an operation affect only this world. A world is either modifiabie or committed (readonly). There are mechanisms to choose a current world, commit a world, and spawn a new world as a child of an existing committed world. The last operation behaves as if it were making a complete copy of the parent database state, but is much more efficient. A world can also be unfrozen if it has no children. A modifiable (leaf) world of the database may be thought of as a "workspace." A person who wishes to modify the database generally selects an existing committed world, creates a modifiable world derived from it, and makes the modifications in the new world. When the changes have reached a stable state, the new world may be committed. Policies and mechanisms for mediating shared access to modifiable worlds are still under study.

Each world has a unique version $I D$, which is a non-empty sequence of positive integers. The root world has ID " 0 ". The ID of the first child of a world $W$ is formed by incrementing the final component of $W$ 's id. Sibling worlds are formed by appending zeros to W's ID. For example, the children of world 1.3.2 would be labeled 1.3.3, 1.3.2.0, 1.3.2.0.0, etc. This numbering is similar to the scheme used by RCS and SCCS, and seems more natural than "Dewey decimal" numbering in the common case of long sequences of 
single-child worlds. For example, a sequence of consecutive derivations from 1.3.2 would yield 1.3.3, 1.3.4, 1.3.5, etc. This numbering scheme can, however, become quite confusing when multiple worlds are derived from the same parent. We expect that worlds will normally be selected by symbolic name or other attributes stored in an index structure (itself stored in the database) rather than by version ID. (This part of the database is still under development.)

In general no changes are permitted in a committed world. However, the value of a derived attribute may be safely deleted and replaced by the special atom "not available." Switching the state of a derived attribute between available and not available is considered a "benign" modification of the database and is permitted in committed worlds.

\subsection{Accessing a CAPITL database}

A CAPITL database can be accessed and manipulated in several ways:

- Directly, through programs written in C++ or Congress.

- Through an interactive X-based browser.

- Through a Unix-compatible interface called EFS.

CAPITL is written in POL, so all of its structures can be accessed as data structures in C+t. For example, nodes are all instances of the class Term, which exports such methods (member functions) as

boolean IsLeaf ();

which enquires whether the term is a leaf, and

Term *Edge (char *selector);

which returns the term referenced by a particular selector (if one exists). Class Integer is a subclass of Term with an IntVal () method that returns its integer value, and so on. Documentation for this interface is currently being written [2].

\subsubsection{Browser}

An interactive browsing interface has been written on top of the $X$ Window System using the InterViews [17] toolkit. The browser supports visiting any object or directory in the object-base and uses typesensitive displays to depict the contents attribute of an object; other attributes are displayed using the Congress expression language (\$2.4). For example, the contents attribute of a source file object is displayed using a text viewer in the Viewing box and any other attributes in the Term View box (see Figure 1). The current focus can be moved to a neighboring object in the naming hierarchy by double clicking an object named in the Object Path or Object Siblings box. The focus can also be changed by typing a path name in the Location Selector box. "Time travel" is accomplished by typing a version ID in the Current World box. Menus exist for creating and destroying objects, invoking the Congress interpreter, and for creating and committing worlds. Multiple simultaneous windows on a database are kept consistent with changes made from any of them.

\subsubsection{EFS}

The CAPITL object-base can be considered an enhanced version of the Unix file system: More types of objects are supported, the set of attributes of an object is extensible, complex relationships among objects can be represented directly, and versioning of the entire database is efficiently supported. However, the differences between the CAPITL object base and the Unix file system interfere with using existing tools. Consider, for example, the problem of compiling a $\mathrm{C}$ source file stored in CAPITL. One approach is to copy the contents of the source object into an ordinary Unix file, invoke the compiler, 


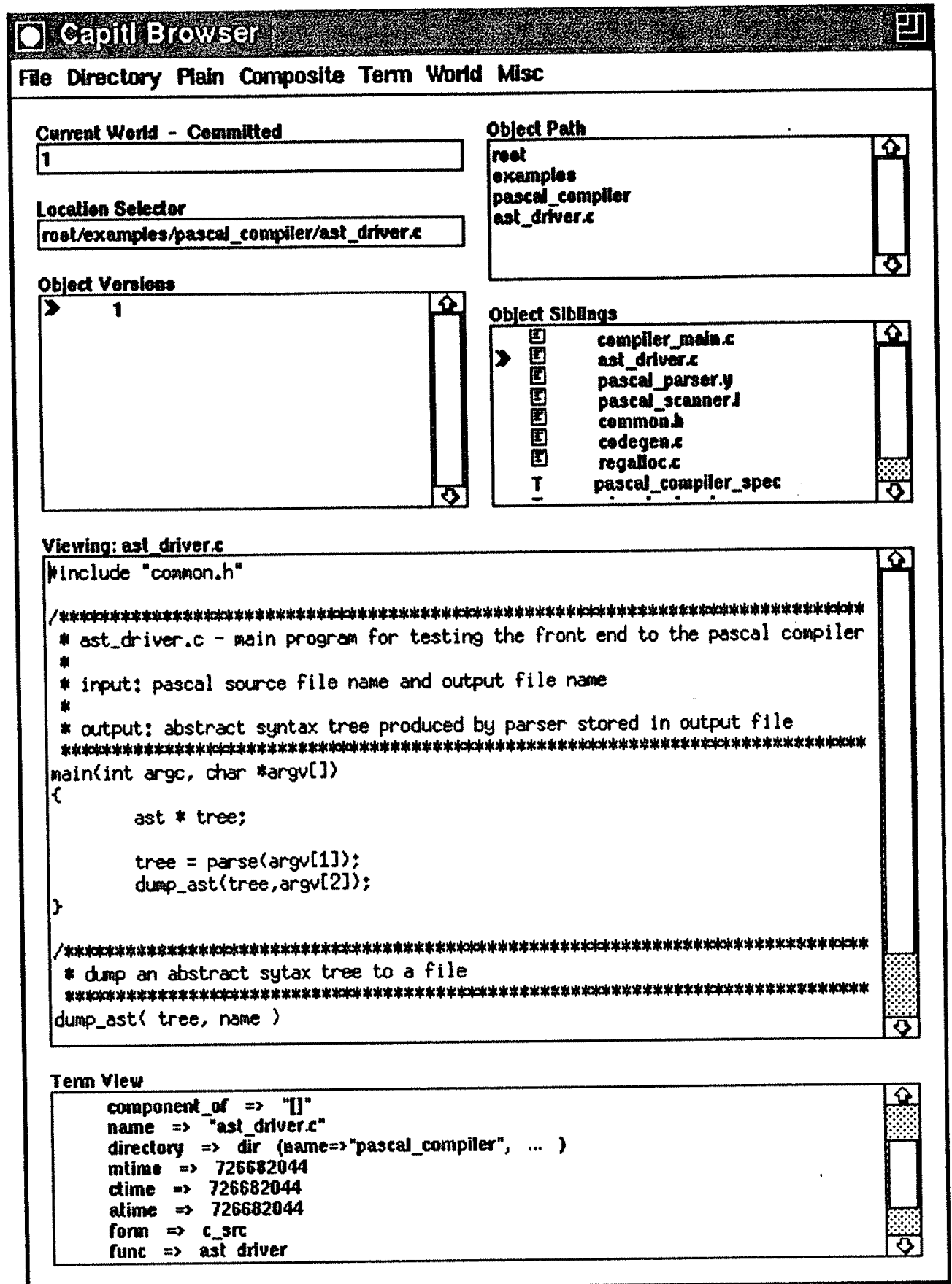

Figure 1

CAPITL Browser

and copy the resulting object module back into CAPITL. A second approach is to store in CAPITL "stub" objects that contain pointers (path names) to Unix files. A third approach is to modify the compiler (perhaps by linking it with alternative versions of the Unix library functions open, read, seek, etc.) so that it can read and modify CAPITL objects. None of these approaches is entirely satisfactory. 
The Emulated File System (EFS) allows programs to access CAPITL objects as if they were Unix files. It is based on the Network File System (NFS) facility [24], which is included in most versions of Unix. NFS was originally designed to support transparent access to remote files. A version of the mount command associates a remote file system with a name, called a mount point in the local file system. System calls that request operations on files below this mount point are forwarded to an NFS server. Normally, the server is the Unix kernel on the remote system, which executes the requests on its local disk. The remote file system thus appears to be grafted into the local name space as a subtree of the mount point. It is possible, however, to designate a user-level process as an NFS server (Figure 2).

The EFS daemon efsd emulates a Unix file system on a CAPITL database. Once a CAPITL database is mounted, its objects can be manipulated by standard system calls (open, read, write, seek, link, stat, etc.) as if they were actual Unix files, directories, and symbolic links. Neither client programs nor the Unix kernel need be modified in any way.

Not all features of the CAPITL database are accessible through EFS. For example, a composite or term object appears to be an empty file from EFS (it behaves like / dev/null). However, EFS does allow access to alternative worlds through an extension of Unix path name syntax. A version ID followed by a colon is interpreted as a request to resolve a path name in a designated world of the database. Path names without version ID's are resolved in a current world analogous to the current working directory. For example,

$$
\text { diff } 3.3: \text { prog.c prog.c }
$$

compares version 3.3 of prog. $c$ with the current version, and

$$
\text { (echo }-n \text { "updates done "; date) } \gg 3.5: \log
$$

adds a line to version 3.5 of $\mathrm{log}$.

As in Unix, a path name that does not start with " $"$ " is interpreted relative to the current directory (and world). Since the Unix kernel uses the same mechanism to resolve chdir requests as open, the shell's cd command can be used to navigate among worlds. For example,

$$
\text { cd 3.2.1: }
$$

sets 3.2.1 as the default world for subsequent file-system requests. The path name supplied in a mount request is interpreted in the same way, so a default world can be specified at mount time, as in

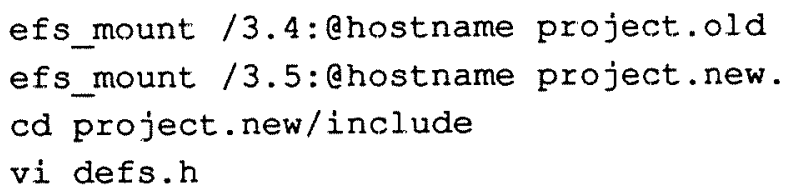

Although EFS was created for CAPITL, it can be used by any C++ system that needs to provide a Unix-compatible interface. It is packaged as a driver program and a set of abstract $\mathrm{C}++$ classes that encapsulate that NFS model. Application-specific classes inherit from these classes. For example, the CAPITL Directory class is derived from both Term and EESdir. Class EFSdir declares methods to add, delete, and lookup directory entries, but supplies no implementation (they are "pure virtual member functions" in C++ terminology). The CAPITL Directory class implements these functions by manipulating the list stored in the contents attribute of the term. More details about the EFS package will be contained in a forthcoming report [26]. 


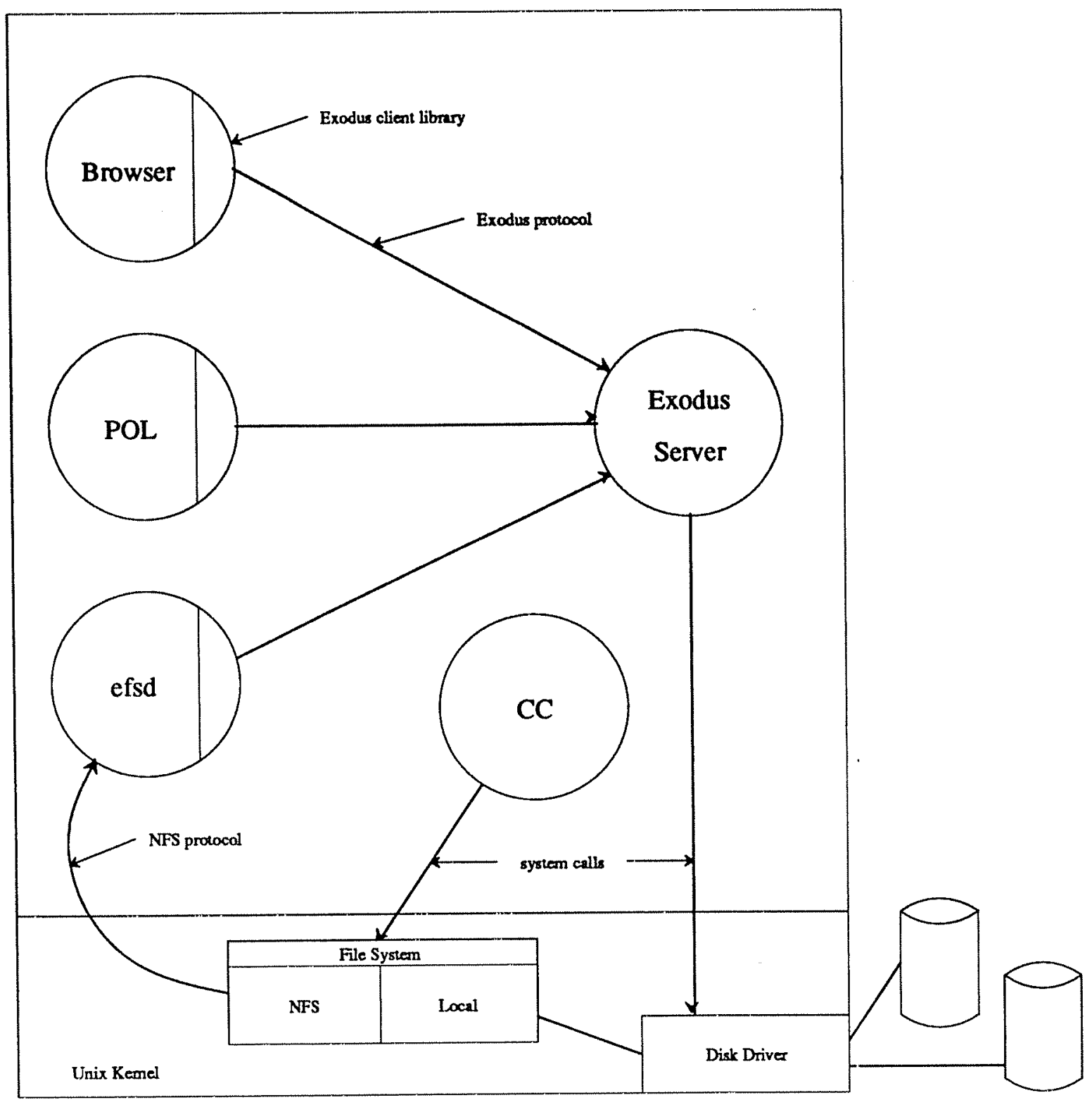

Figure 2

\section{CAPITL Process Architecture}

\section{Software Configuration Management in CAPITL}

CAPITL provides assistance for constructing and maintaining software products. A product is the output of a series of tools applied to a set of objects. The goal is to produce a "correct" version of a product as efficiently as possible. Correctness is a fuzzy concept that has possibly different meanings in different situations. Therefore, CAPITL supports a powerful constraint language (POL) allowing system designers to describe attributes and integrity constraints as appropriate to each application. CAPITL guarantees that all derived objects are correctly created by applying tools to inputs in accordance with these specifications. In addition, CAPITL ensures that all products are current ( $i e$ none of the objects used to 
create the product has changed since the product was constructed). When building (or rebuilding) a product CAPITL speeds up the process by reusing previous work when possible.

CAPITL software objects are classified as source or derived. A source object is created by a human being or by some other process outside the control of CAPITL. A derived object is created by applying a tool object to an input object. Each tool has exactly one input, which can be a set cr list of other objects. (Construction of such composite objects is described more fully below.) The tools as well as inputs can be either source or derived. Each derived object can be viewed as the value of a derivation-an expression tree whose leaves are source objects and whose interior nodes represent occurrences of a built-in apply operator. Objects are all represented in CAPITL as POL terms, and thus can have a variety of attributes. Some of these attributes constrain the set of well-formed derivation expressions (\$4.1). In particular, each tool object has an in attribute that must match the object to which it is applied, and an out attribute that constrains the attributes of the result. Since the attributes are terms that can contain variables, an object can be partially specified. An incomplete object can be more fully specified by replacing occurrences of variables with other terms. ${ }^{6}$ An object with an unspecified contents is called an abstract object.

A user requests the derivation of a new object by constructing an abstract goal object and invoking the planner (\$4.4) and the builder (\$4.5) which are Congress programs supplied with CAPITL. The planner determines how to create objects and supplies them with code attributes, while the builder creates the contents of planned objects by evaluating expressions in their code attribute. The code attribute of an object is a list of alternative build expressions, each of which contains references to a tool object and an input object. When asked to plan an object without a code attribute, the planner searches the database for tools and matching inputs such that the result of applying the tool to the input yields the desired goal. It then recursively plans the tool and the input.

The planner can infer the need to construct new derived objects. If it finds a wol capable of creating a desired goal object but no corresponding input, it tentatively creates an abstract object representing the input and plans it. If the input is a composite object (for example, a list of inputs to the linkage editor), the planner searches for a template ( $\$ 4.2)$ that describes potential collections. The planner can also create new tool objects. A tool description ( $\$ 4.3)$ contains an input/output description of a potential tool. If the planner fails to find a tool that can create a goal object but discovers an appropriate tool description, it will instantiate the description as a new tool object and attempt to plan it. For example, the planner may fail to find a cross-compiler capable of building an object module for a particular machine architecture, but a tool description may suggest that such a compiler can be built from existing sources.

The builder completes the process of making an abstract goal concrete by executing one of the build expressions in its code attribute. If the build is successful, the result is placed in the contents, and a record of the specific tool and input used to create it is placed in the provenance attribute of the derived object. If it is unsuccessful (for example, if the tool is a compiler that discovers an error in its input), the build expression is marked as "failed," the planner is invoked to find other altematives, and another build expression is tried.

\footnotetext{
${ }^{6} \mathrm{~A}$ consequence of the definition of unification in $\$ 2.4$ is that the absence of an atribute is equivalent to an attribute whose value is a variable.
} 


\subsection{Object Descriptions}

The attributes code, contents, provenance, form, functionality, and references are used by CAPITL for the purpose of planning and building. The first three of these have been briefly described above. The attributes form and functionality jointly describe the "type" of an object. These attributes control the matching of tools to inputs and outputs. The references attribute is used to record extra dependencies among objects.

4.1.1. Form The form of an object is its type when used as an argument to a tool. In the simplest case the form attribute is a simple atom with an application-defined meaning, such as c_source or object_code. More detailed information can be specified by more complicated terms. For example, the form attribute of an object module might be specified as

$$
\text { object_code ( opt } \Rightarrow \text { no, debug_symbols } \Rightarrow \text { yes) }
$$

Since forms are terms, the partial order induced by unification can be used to express subtype relationships. For example, object_code (opt $\Rightarrow$ no, debug_symbols $\Rightarrow$ yes) is compatible with (unifies with) object_code. The planner interprets two values for the form attribute as having special meaning: The value $\operatorname{bag}(T)$ is interpreted as a homogeneous multi-set of type $T$, and the value record $\left(T_{1}, \cdots, T_{n}\right)$ as a heterogeneous collection of types $T_{1}$ through $T_{n}$. All other values are interpreted as non-structured (atomic) types.

4.1.2. Functionality The functionality attribute of an object is a description of what the object does. For tool objects, the functionality attribute is the type signature of the tool and describes its behavior via an in/out pattern. For example, the functionality of a $\mathrm{C}$ compiler might be specified by the term

$$
\begin{aligned}
\text { func (in } & \Rightarrow \text { obj(form } \Rightarrow \text { c_source, functionality } \Rightarrow F \text { ), } \\
\text { out } & \Rightarrow \text { obj(form } \Rightarrow \text { object_code, functionality } \Rightarrow F \text { )), }
\end{aligned}
$$

which states that the input must be $C$ source code, the output will be object code, and the functionality (semantics) of the module is preserved.

4.1.3. Contents, Provenance, and Code For atomic objects the contents attribute is uninterpreted by CAPITL. For composite objects the contents is the set of sub-objects that comprise the object. The provenance of a derived object specifies the tool and input used to create the contents. Since the provenance names specific objects (by their $\mathrm{OID}$ ), it is completely synonymous with the contents, in the sense that the same contents could be recreated by rerunning the same sol on the same input. The code attribute is a set of expressions, each of which is type-compatible with the object and is thus a potential recipe for generating the contents; it represents a non-deterministic program for creating the contents. The code, provenance, and contents are in some sense all manifestations of the same value where the code is the least specific and the contents the most specific.

4.1.4. References The references attribute identifies objects that are referred to inside an atomic object's contents. It can be an assertion from the specification writer or the output of some languagespecific tool. For example, suppose a $\mathrm{C}$ source file has many "\#include" preprocessor directives. If the $\mathrm{C}$ compiler and preprocessor are being modelled as one tool, the references attibute would represent the set of include files required to run the compiler/preprocessor tool. These dependencies are needed for maintaining consistency after changes. References are used in checking whether derived objects are upto-date. They are also useful for browsing source objects. 
When describing an object, a specification writer has two ways to refer to other objects. A generic reference is a pattern that matches other objects in the object-base. For example, the term

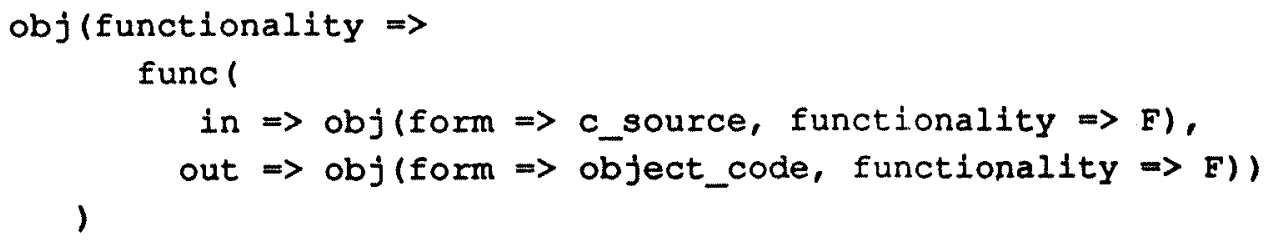

represents a generic reference to any $\mathrm{C}$ compiler. If the environment contained two $\mathrm{C}$ compilers, such as " $c c$ " and "gcc", either could match. A specific reference defines a unique object. A specific reference can be created from a generic reference by adding enough other attributes to make the reference unique or by using the object's identity. Because it is difficult to guarantee that a set of attributes always identifies exactly one object, the identity of an object is preferred for specific references. For example, the provenance of an object module might contain tool $\Rightarrow G C C$ where Gcc is a tag for a specific compiler object.

\subsection{Templates}

A system is a collection of objects that when combined form a meaningful "chunk". Systems are described in CAPITL by specially formed objects called templates. (Such descriptions are often referred to as system models.) A template consists of a set of generic references, specifying (at least) the functionality of each sub-object, and a set of constraints. The constraints are used to specialize the generic references prior to instantiating the template. Templates are instantiated by making a copy of the template, calling all the predicates listed in the constraints, and then resolving each generic reference via a database lookup. The result is an object that unifies with the template, but has all the generic references in its contents resolved into specific references. Because template instantiations are objects, they can be used as the subparts of other template instantiations. Thus, system descriptions in CAPITL can be composed.

\subsection{Tool Descriptions}

CAPITL uses separate tool descriptions to specify the available set of tools. Tool behavior (as opposed to tool objects) is described via specially formed Congress rules stored in a separate rule-base that can be imported into a Congress program. Tool descriptions serve two purposes. First, the presence of a tool description alerts the planner that it is possible to build a tool with a particular functionality. Hence tool generators can be modelled in CAPITL. Second, tool descriptions allow more complicated descriptions of tool behavior than is possible with simple input/output signature patterns. Section 5.2 contains an example that illustrates this feature.

\subsection{Planning}

Planning is the process of finding a set of source objects and tool applications needed to satisfy a request. The planner makes use of three kinds of data: existing planned objects, template objects, and tool descriptions. The planner searches the space of all possible "well formed" tool and object combinations for expressions whose results match the goal and that only contain references to atomic objects or fully specified composite objects. These expressions are stored in the code attribute of an object and represent a potential recipe for constructing the contents of that object-the planner does not guarantee that an expression will succeed when evaluated. During planning, templates are instantiated and any constraints attached to objects are checked ensuring that all objects used to build a system conform to the constraints specified in the description of the system. Such constraints can easily encode certain kinds of semantic 
correctness such as "use all debugging versions", by forcing the appropriate sub-types to be used for derived objects (and hence forcing the use of tools that produce debugging information).

The planner avoids repeating work by keeping track of the current state of an object's plan with an additional plan_state attribute. When the planner creates a new object to represent an intermediate result, its state is attempted. If a build expression is found, the state changes to successful, otherwise it changes to failed. Using this attribute the planner can avoid attempting to plan an object that has already failed and avoid derivation loops, which occur if an object is needed in order to derive its own contents.

Planning may be computationally expensive because it exhaustively searches a potentially exponential space. To speed the planning process we are experimenting with lazy generation of expressions and better search strategies. Instead of deriving all equivalent expressions, our prototype stops as soon as a single expression is found. (A "replan" request is available to search for additional expressions.) Currently, the planner uses a blind depth-first search. By ranking choices, perhaps by using approximate tool costs, a branch-and-bound strategy could be used to improve performance.

\subsection{Building}

Given a reference to a derived object, the job of the builder is to make the contents, provenance, and code attributes consistent. They are consistent if the provenance is one of the expressions in the code and it evaluates to the contents.

The builder traverses the expression graph defined by the code and provenance attributes. Depending on the state of an object there are three cases that arise:

(1) The object does not have a provenance or contents attribute. The builder evaluates the first untried expression in the code. If the expression is successful, the builder stores that expression as the provenance of the object. If the expression fails (perhaps due to a syntax error in a source program), the builder annotates that expression as unsuccessful and invokes the planner, which attempts to find a different expression for the object. If the planner is successful, the build continues.

(2) The object has a contents (and hence a provenance). In this case the builder must determine if the contents is still valid. In a committed world, the contents must be the value of the expression stored in the provenance, so no reconstruction is necessary. In a mutable world, objects referenced by the provenance may have changed since it was last evaluated. To determine if the contents is still up to date, the builder uses timestamps. The mtime attribute of an object records the time its contents was last modified. Associated with the provenance of a derived object is a provenance_timestamp that records the timestamps of the tool and argument. If the timestamps match, the contents is still valid. Otherwise, the provenance is re-evaluated. A more precise notion of validity that relies on semantic properties of the objects involved could be used, potentially allowing fewer expressions to be evaluated [28].

(3) The object has a provenance but no contents, indicating that a user conserved space by deleting the contents. The contents can be regenerated by simply evaluating the provenance.

\subsection{Discussion}

Separating the planning and building phases has several advantages. The decomposition of a system into sub-systems tends to change slowly, allowing the output of the planner to be used many times. Hence 
the cost of planning is amortized over many builds. Moreover, the separation simplifies the builder: It is only concerned with equivalence between a functional expression and a "cached" copy of the result of that expression; the planner does all the work of selecting objects and tools.

One can view the planner as a code generator and the builder as a code evaluator. To increase the speed of building a separate optimize phase could be used after planning to perform traditional compiler optimizations such as strength reduction and elimination of intermediate values. These optimized expressions would then be saved in the provenance for future use. For example, the expressions produced by the planner uses an object to hold the result of every tool application. A linear sequence of tool applications in which the intermediate results were not specifically requested could be compressed into a single pipeline invocation.

CAPITL worlds provide a means to group objects with similar semantic properties. This version mechanism assists planning by limiting the search needed to construct a product. Because only one version of an object is visible in a given world, the planner does not need to choose among the (potentially large) set of versions of each source object. Hence the combinatorial explosion associated with combining components represented as version sets is avoided during planning.

\section{Examples}

To illustrate the concepts of the previous section, we present two examples of simple subsystems. The first example illustrates how an executable program is built from sources in a variety of languages. The second example is drawn from the domain of document processing.

\subsection{A Pascal Program Analyzer}

The first example is a simple program analyzer that translates Pascal source fi.es into abstract syntax trees (AST's). Such an analyzer might be a component of a compiler or other larger system. We assume that four source objects are available: a Lex [16] specification of tokens, a YACC [9] specification of a grammar, a driver program written in $\mathrm{C}$, and a common file of declarations included by all three sources. These objects are shown in Figure 3. Cormon is the file of common declarations. It has only two attributes, a format (source for the $\mathrm{C}$ preprocessor) and the actual text contents. Main is the main program. Its format is $\mathrm{C}$ source, it depends on Common, and its functionality (semantics) is described by the atom "ast_driver." The Lex and YACC source objects are similar. The references attributes might be supplied manually by the author of the program or it might be deduced by a tool such as the Unix makedepend utility. The functionality attributes would be supplied manually by the designer of the analyzer package.

Pascal_Analyzer describes the functionality of the desired tool: It should translate a Pascal source object into an abstract syntax tree preserving its functionality. Analyzer_spec is a template that specifies how the component functionalities ast_driver, pascal_parser, and pascal_scanner can be assembled to produce a tool that translates Pascal source into abstract syntax trees. Analyzer_Spec may be thought of as a "tool" that produces a composite object (a package of objects) from components. This specification conveys three pieces of information: First, that the functionality Pascal_Analyzer is the sum of the functionalities ast_driver, pascal_parser, and pascal_scanner; second, that resulting object has form bag $(T)$, where $T$ is the form of each component; and third, that all the components should have a property called debug_level. For example, if it desired that all object files have the debugging property, the debug_level predicate would be defined as 


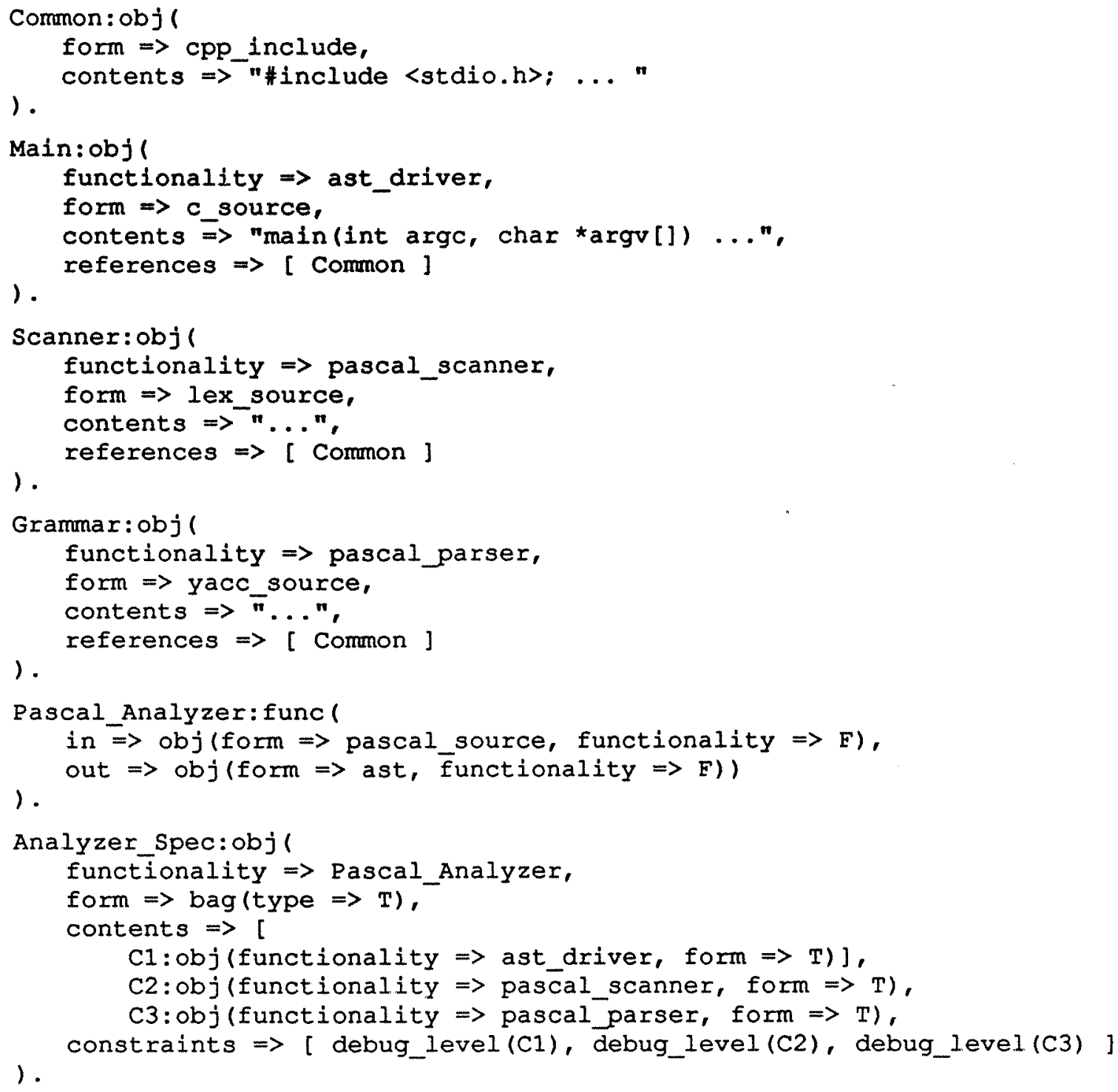

Figure 3

\section{Source Objects}

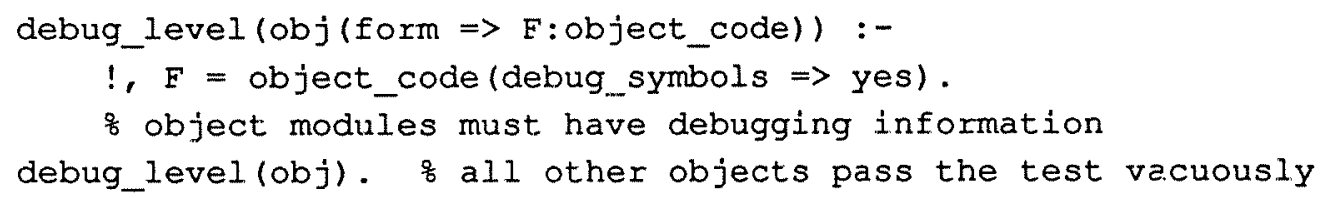

Figure 4 shows a variety of tool specifications. The tool specification Lex_spec describes the functionality of a Lex processor-it transforms a lex_source input into c_source preserving semantics. The object Lex is an executable program that conforms to this specification. Similarly, each of other tools would have a corresponding tool description. Since each tool description is identical to the input/output signature of the corresponding tool, we omit the remaining descriptions. We have chosen to 
model the debugging and optimizing versions of the $\mathrm{C}$ compiler as two distinct tools.

Calling the planner with the goal

$$
\text { obj ( form } \Rightarrow \text { executable, functionality } \Rightarrow \text { Pascal_Analyzer ) }
$$

will create a partial object with the desired functionality and form. If planning is successful, the object will contain a build expression in its code attribute that can be used to create its contents. The planner will also create partial objects for intermediate objects as shown in Figure 5.

At this point, the builder may be invoked on the object Goal. Assuming there were no errors, the builder would fill in the contents and provenance attributes of each object in Figure 5.

Suppose the state of the system is frozen by committing the current world and a new one is created. Consider two different kinds of modifications to the system:

(1) The only action in the new world is to modify the contents of Scanner. In this scenario, the same plan can be reused (in fact calling the planner will result in no changes), and the builder will reuse ParserObj and MainObj, rebuilding only ScannerC, ScannerObj, and Goal.

(2) No sources are modified, but an optimized version of the analyzer is desired. The definition of the Congress predicate debug_level is changed to

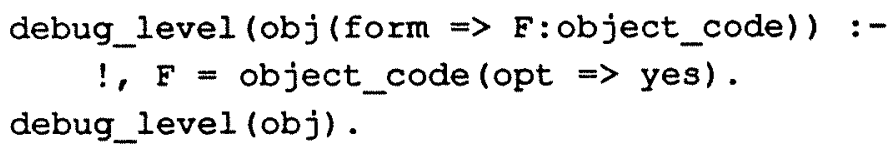

There are two choices for where to store the new version: in a new object distinct from the existing debugging version or in the existing analyzer object (replacing the debugging version in this world). In the latter case, the code attribute would need to be cleared before planning (if not, the object would fail the current set of constraints and would fail to be used). In either case the planner would be re-invoked on the appropriate goal object. The existing objec: Object_Modules would not be used because its constraints fail with the current definition of debug_level. Instead, the planner would create a new composite object from the Analyzer_Spec template

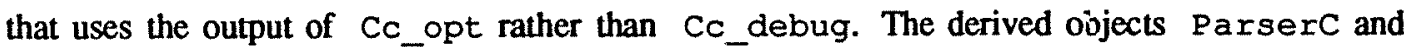
Scannerc from the previous world would be used without modification.

\subsection{Document Processing}

The Unix troff document processing system includes a variety of special-purpose preprocessors. If a document does not use a particular feature, the corresponding preprocessor need not be applied. For example, eqn only needs to be run on documents that contain mathematical equations, while $t b l$ is only required for documents containing tables. We could model these tools by defining a variety of types as in the previous example, defining eqn to be a translator from troff_with_eqn to troff, etc. However, this approach would require a different type for each subset of the features. A better approach defines one form, troff, with subtypes for different sets of required features. For example a document with equations and tables would have form troff (features $\Rightarrow$ [eqn, tbl]). The tool description for eqn would then be

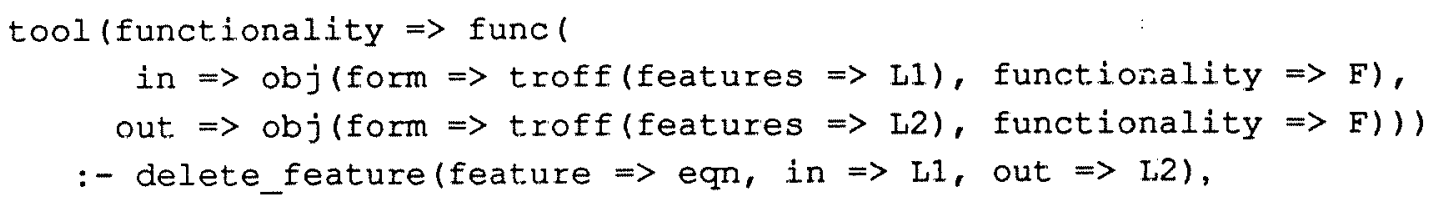

where the predicate delete_feature searches list L1 for an occurrence of eqn and removes it. If a 


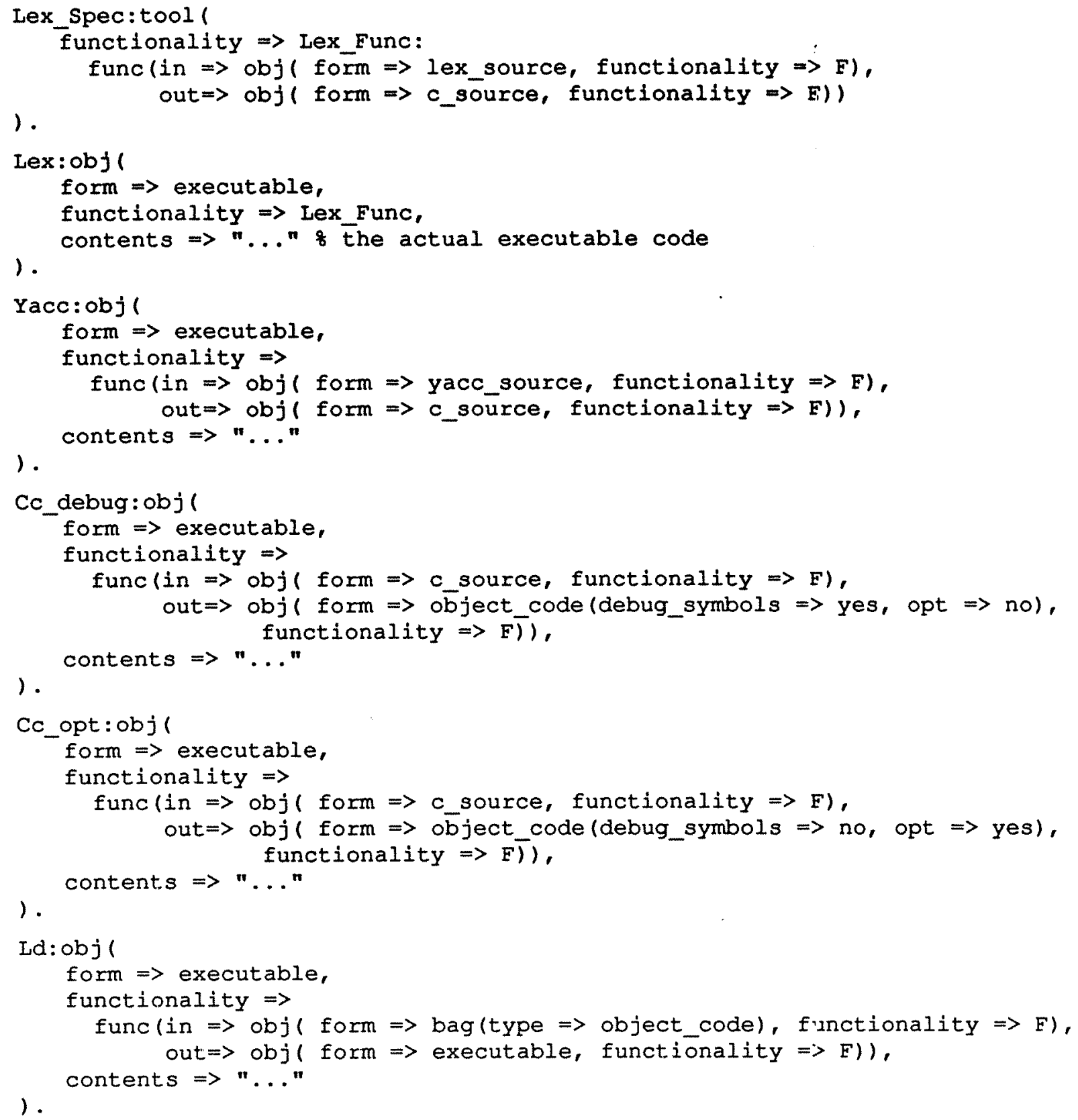

Figure 4

\section{Tool Objects}

document does not use eqn the body of this rule will fail and the planner will not consider it applicable. A more sophisticated version of delete feature can encode the requirement that some processors have to be run before others. As with the references attribute, the features of a troff document could be added manually or by a processor that analyzes a document. 


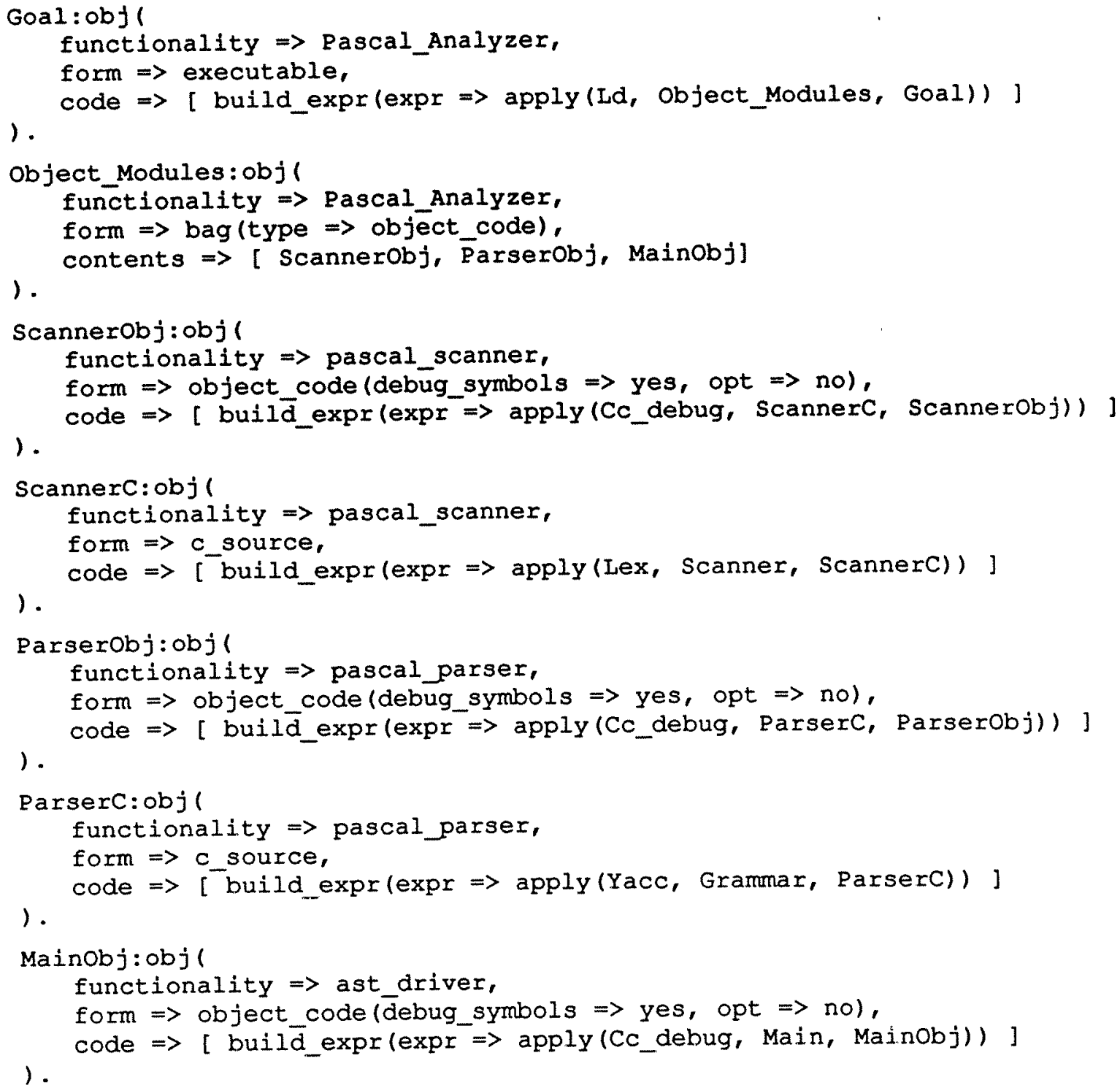

Figure 5

\section{Derived Objects After Planning}

\section{Related Work}

Many of the ideas in the design of CAPITL are present in other systems. CAPITL's main distinguishing feature is tight integration of a process programming language (Congress) with an underlying versioned, object-oriented database. A software object is not just described by a Congress expression, it is a Congress expression. Congress is declarative rather than procedural; it allows system designers to concentrate on the properties of objects and subsystems rather than on procedures to manipulate them.

All of the following systems provide good support for building; they differ primarily in how systems are specified, how version selection is accomplished, and what kinds of consistency are guaranteed. 
DSEE. DSEE $[14,15]$ is a commercial environment that manages software in a network of distributed (Apollo) workstations. It supports a notion of "time travel" by compactly storing versions of source files and providing a tool, the History Manager, that associates symbolic attributes with particular versions. A separate tool, the Release Manager, maintains groups of consistent files. DSEE configurations start with a user-supplied dependency relation called the system model. Version selection rules are used to bind object references in the system model to specific versions in the file system. DSEE supports many other features needed in a distributed environment and is one of the most advanced systems available commercially.

Shape. Shape $[18,19]$ integrates Make with a version control system similar to RCS. Shape is backwards compatible with Make and adds version selection rules comparable to those in DSEE. These rules use regular expressions to specify an ordered list of version preferences, such as "use the newest version of all components I am working on", and "use the newest stable version of all other components." The default selection rule is to use the most recent version of all components as in Make. Shape stores source objects in an attributed file system and can distinguish different versions of objects by using "version attributes." The ability to use existing Makefiles is convenient, but such files contain a static description of the system, and maintaining that description becomes more and more difficult as the system grows larger. Like DSEE, Shape relies on an external tool for checking that a configuration is consistent.

Odin. Odin [4] is a system for integrating existing tools into a single environment. Tools are described declaratively and then linked into a derivation graph that summarizes all the "type correct" derivations possible given the current set of tools. The derivation graph is then used to infer build steps given a request for a particular object. As with Make, the programmer must specify all the dependencies of a configuration. Version selection and consistency are not integrated into the system. From a practical point of view, Odin's ability to use existing tools is appealing; our use of EFS achieves the same functionality.

Jason. Jason is a generic software configuration system [31] that constructs a software environment from a given set of parameters. These parameters include class definitions (object schemas), consistency constraints, and build plans (dependency relations). Created environments can later be extended, but because of the "compiled" nature of generated environments, such extensions are limited to additions and refinements. This limitation prevents Jason from supporting certain kinds of evolution (such as reorganizing a two-component system into a three-component system). Jason uses a powerful constraint language (full first-order predicate calculus) and compiles the given constraints into procedures that check the consistency of configurations. A rigorous algebraic model provides Jason with a strong theoretical foundation not present in most other systems.

Adele/Nomade. Adele [6-8] (and its successor Nomade [8]) is a constraint-based environment for SCM. Constraints are quantifier-free boolean expressions and are used to infer consistent configurations, including the dependency relation between components. The advantage of this approach is that programmers specify what they want and the system figures out how to produce it. Our system also contains this general concept, but our use of Congress allows more powerful constraints.

SMILE/Marvel. SMILE and Marvel [10-12] are two rule based environments that emphasize support for the edit/debug/build cycle. Their goal is to provide a "fileless" environment for programmers by making the environment responsible for invisibly maintaining derived objects. Their rules use Hoare-style pre and post conditions to trigger actions and can be used for either forward-chaining or backward-chaining inferences. Forward-chaining corresponds to opportunistic computation and backward-chaining corresponds to the method employed by traditional build tools such as Make. 


\section{Status and Future Plans}

A prototype of CAPITL has been implemented that includes all the basic components: object-base, worlds, EFS, Congress compiler, POL interpreter, browser, planner and builder. The planner and builder subsume the functionality of Make. We have tested them on small examples with atout 10 source objects, 20 tool descriptions and 50 objects total. The planner correctly generates plans, the builder constructs executable binaries (running tools such as the $\mathrm{C}$ compiler with the aid of EFS), and the resulting programs can be invoked through the interactive user interface. After changes, the builder is able to rebuild targets taking advantage of unchanged sources and intermediate objects.

Our next goal is to use CAPITL to control its own development. Although POL and CAPITL cannot be considered "large" (they consist of about 32,000 lines of $\mathrm{C}++$ code and 3000 lines of Congress code), the experience should provide feedback on whether the ideas in CAPITL scale to larger systems. Most of the required functionality is in place. The principle obstacles are performance limitations, and some rough spots in the user interface.

\subsection{Performance}

The planner and builder are currently an order of magnitude slower than Make. The main thrust of our current work is to identify and eliminate performance bottlenecks. Two modifications mentioned earlier should help: an optimizer for build expressions and a better search strategy for the planner. Indexes can be used to improve database searches, and critical parts of the POL interpreter can be hand tuned. EFS is considerably slower than native or NFS file system access, particularly for writing, so tool invocations (such as compiling) are severely degraded. The main source of the problem appears to be inappropriate timeouts and buffering strategies in the NFS client code in the Unix kemel. A successor to Exodus, called Shore, is being developed by a separate project; one of the goals of Shore is to provide a facility similar to EFS with higher performance.

\subsection{User Interface}

Several enhancements to the user interface are needed to make CAPITL usable by system architects and developers.

Syntactic Sugar. Currently, source objects, tool descriptions, templates and goals are all created "by hand" as Congress expressions. Forms-based interfaces would help to streamline this process and eliminate the need for developers to learn about logic programming. The browser understands a few attributes and has special displays for them (for example, the contents of a plain file object is displayed in a text window), but most attributes are simply unparsed into the Congress expression language and minimally pretty-printed. More special-case displays would help.

Idioms. Common terms such as frequently used forms and functionalities (such as the subterm Lex_Func in Figure 4) could be collected in libraries and displayed by the graphical interface as icons for pasting into new descriptions. They can also serve as the basis for creating new types through subtyping.

Worlds. Currently a user must set the default world either by navigating the tree of worlds, or by explicitly typing version ID's as sequences of integers. We intend to introduce "world objects" so that mnemonic names and other attributes can be associated with worlds. 


\subsection{Additional Functionality}

The user interface enhancements suggested above are all fairly straightforward. Other usability enhancements require more fundamental research.

Version Management. Versioned worlds are simple to understand, but policies for managing them need to be explored. CAPITL contains no provisions for mediating access to mutable worlds except that provided by Exodus, which only serializes simple updates; more sophisticated kinds of long-term locks are needed. Mechanisms for selecting worlds and maintaining their internal consistency need to be developed. Tools are needed to support an "algebra" of modifications that allow modifications to be added and subtracted. An example of "addition" is reconciling and merging concurrent updates that created sibling worlds. An example of "subtraction" arises when two updates were applied in sequence, and it is desirable to generate the world that would have arisen if the second update were applied but not the first.

Cache Management. A derived attribute is one whose value is an immutable function of other attributes. Derived attributes can be elided or recomputed even in a committed world. Currently, only the contents attribute is a derived attribute (as a function of the provenance), and it is implemented in an ad hoc fashion in the builder. We would like to extend the idea in a uniform way to other attributes. The contents of a derived object must currently be deleted by hand. A cache management tool that selectively flushes cached attributes based on their size, time since last use, and cost of reconstruction, would be useful. More generally, all derived objects may be thought of as a residing in a cache. In some cases there may be more than one equivalent way to build a product. For example, an executable program might be rebuilt from its sources or reconstituted from a compressed version. Thus cache maintenance is intimately tied to the larger issue of planning.

\section{Acknowledgements}

Many people have helped to bring CAPITL to its current state. S. T. S. Prasad was the first to explore the application of Prolog to configuration management. Tony Rich carried this work forward. His thesis is the basis for many of the ideas in Section 4 of this paper, and the current planner and builder owe much of their content to his work. Odysseas Tsatalos, Theoharis Hadjiioannou, and Trip Lazarus have made substantial contributions to the $\mathrm{X}$-based interactive interface. Lazarus also implemented the worlds mechanism and integrated EFS into CAPITL. Delta files were implemented by Tsatalos. Tom Ball and Sam Bates offered many helpful comments on earlier drafts of this paper.

\section{References}

[1] H. Aït-Kaci and R. Nasr, LOGIN: A Logic Programming Language with Built-In Inheritance, Journal of Logic Programming, Mar. 1986, 181-215.

[2] P. Adams and M. Solomon, POL: Persistent Objects with Logic, Submitted for publication.

[3] M. Carey, D. DeWitt, G. Graefe, D. Haight, J. Richardson, D. Schuh, E. Shekita and S. Vandenberg, The EXODUS Extensible DBMS Project: An Overview, in Readings in ObjectOriented Databases, S. Zdonik and D. Maier (ed.), Morgan-Kaufman, 1990.

[4] G. Clemm and L. Osterweil, A Mechanism for Environment Integration, ACM Trans. Prog. Lang. and Systems 12, 1 (Jan. 1990), 1-25.

[5] J. R. Driscol, N. Sarnak, D. D. Sleator and Targan, Making Data Structures Persistent, Journal of Computer and System Sciences 38, 1 (Feb. 1989), 86-124. 
J. Estublier, S. Ghoul and S. Krakowiak, Preliminary Experience with a Configuration Control System, Proceedings of the Software Eng. Notes/SIGPLAN Notices Software Eng. Symposium on Practical Software Development Environments, Apr. 1984, 149-156.

[7] J. Estublier, A Configuration Manager: The ADELE Database of Programs, Proceedings of the Workshop on Software Engineering Environments for Programming-in-the-Large, Harwichport, MA, June 1985, 140-147.

[8] J. Estublier, Configuration Management: The Notion and the Tools, Proceedings of the International Workshop on Software Version and Configuration Control, Grassau, W. Germany, Jan. 27-29, 1988, 38-61.

[9] S. C. Johnson, YACC-Yet Another Compiler Compiler, C. S. Technical Report \#32, Bell Laboratories, Murray Hill, NJ, 1975.

[10] G. Kaiser and P. H. Feiler, SMLE/MARVEL: Two Approaches to Knowledge-Based Programming Environments, Tech. Report CU-CS-227-86, Department of Computer Science, Columbia University, New York, NY 10027, Oct. 1986.

[11] G. Kaiser and P. H. Feiler, Granularity Issues in a Knowledge-Based Programming Environment, 2nd Kansas Conference on Knowledge-Based Software Development, Manhattan, KA, Oct. 1986.

[12] G. Kaiser and P. H. Feiler, An Architecture for Intelligent Assistance in Software Development, Proceedings of the Ninth International Conference on Software Engineering, Monterey, CA, Mar. 1987, 80-88 .

[13] K. Knight, Unification: A Multidisciplinary Survey, ACM Computing Surveys 21, 1 (Mar. 1989), 93-124.

[14] D. B. Leblang and R. P. Chase, Jr., Computer-Aided Software Engineering in a Distributed Workstation Environment, SIGPLAN Notices Notices 19, 5 (Apr. 1984), 104-112 .

[15] D. B. Leblang and G. D. McLean, Jr., Configuration Management for 'Large-Scale Software Development Efforts, Proceedings of the Workshop on Software Engineering Environments for Programming-in-the-Large, Harwichport, MA, June 1985, 122-127.

[16] M. E. Lesk, Lex - A lexical analyzer generator, C. S. Technical Report $¥ 39$, Bell Laboratories, Murray Hill, NJ, October 1975.

[17] M. A. Linton, J. M. Vlissides and P. R. Calder, Composing User Interfaces with InterViews, IEEE Computer, February 1989, 8-24.

[18] A. Mahler and A. Lampen, Shape-- A Software Configuration Management Tool, Proceedings of the International Workshop on Software Version and Configuration Control, Grassau, W. Germany , Jan. 1988.

[19] A. Mahler and A. Lampen, An Integrated Toolset for Engineering Software Configurations, Proceedings of the ACM Software Eng. Notes/SIGPLAN Notices Software Engineering Symposium on Practical Software Development Environments in SIGPLAN Notices Notices 24, 2 (Feb. 1989), 191-200.

[20] J. K. Ousterhout, Tcl: An Embeddable Command Language, 1990 Winter USENIX Conference Proceedings, 1990.

[21] A. Rich and M. Solomon, A Logic-Based Approach to System Modelling, Workshop on Software Configuration Management, Trondheim, Norway, June 1991, \&4-93. 
[22] A. Rich, Logic-Based System Modelling, PhD Thesis, University of Wisconsin-Madison, Aug. 1991.

[23] J. Richardson, M. Carey and D. Schuh, The Design of the E Programming Language, $A C M$ Trans. Prog. Lang. and Systems, to appear.

[24] R. Sandberg, D. Goldberg, S. Kleiman, D. Walsh and B. Lyon, Design and implementation of the Sun Network filesystem, Proceedings of the Summer 1985 USENIX Conference, Portland, OR, June 1985, 119-130.

[25] R. W. Scheifler and J. Gettys, The X Window System, ACM Transactions on Graphics 16, 8 (Aug. 1983), 57-69.

[26] M. Solomon, EFS: The Extensible File System, University of Wisconsin Technical Report, In preparation.

[27] S. M. Sutton, Jr., D. Heimbigner and L. J. Osterweil, Managing Change in Software Development through Process Programing, University of Colorado at Boulder tech report \#CU-CS531-91, June 1991.

[28] W. F. Tichy and M. C. Baker, Smart Recompilation, 12th Annual ACM Symposium on Principles of Programming Languages, New Orleans, Louisiana, Jan. 14-16, 1985, 236-244.

[29] W. F. Tichy, RCS: A System for Version Control, Software-Practice and Experience 15, 7 (July 1985), 637-654.

[30] W. F. Tichy, Tools for Software configuration Management, Proceedings of the First International Workshop on Software Version and Configuration Control, Grassau, FRG, Jan. 1988, 120.

[31] D. Wiebe, Generic Software Configuration Management: Theory and Design, (Thesis) University of Washington Tech Report \#90-07-03, 1990. 